\title{
A Dynamic Data Driven Wildland Fire Model
}

\author{
Jan Mandel ${ }^{1,2}$, Jonathan D. Beezley ${ }^{1,2}$, Lynn S. Bennethum ${ }^{1}$, \\ Soham Chakraborty ${ }^{3}$, Janice L. Coen ${ }^{2}$, Craig C. Douglas ${ }^{3,5}$, Jay Hatcher ${ }^{3}$, \\ Minjeong $\mathrm{Kim}^{1}$, and Anthony Vodacek $^{4}$ \\ ${ }^{1}$ University of Colorado at Denver and Health Sciences Center, Denver, CO \\ 80217-3364, USA \\ ${ }^{2}$ National Center for Atmospheric Research, Boulder, CO 80307-3000, USA \\ ${ }^{3}$ University of Kentucky, Lexington, KY 40506-0045, USA \\ ${ }^{4}$ Rochester Institute of Technology, Rochester, NY 14623-5603, USA \\ ${ }^{5}$ Yale University, New Haven, CT 06520-8285, USA
}

\begin{abstract}
We present an overview of an ongoing project to build DDDAS to use all available data for a short term wildfire prediction. The project involves new data assimilation methods to inject data into a running simulation, a physics based model coupled with weather prediction, on-site data acquisition using sensors that can survive a passing fire, and on-line visualization using Google Earth.
\end{abstract}

\section{Introduction}

DDDAS for a short-term wildland fire prediction is a challenging problem. Techniques standard in geophysical applications generally do not work because the nonlinearity of fire models are much stronger than those of the atmosphere or the ocean; data is incomplete; and it is not clear which model is the best for physical representation and faster than real time speed.

\section{$2 \quad$ Fire Model}

The goal in wildland fire modeling is to predict the behavior of a complex system involving many processes and uncertain data by a physical model that reproduces important dynamic behaviors. Our overall approach is to create a mathematical model at the scales at which the dominant behaviors of the system occur and the data exist.

Perhaps the simplest PDE based wildland fire model [2] is of the form

$$
\begin{aligned}
& \frac{d T}{d t}=\nabla \cdot(k \nabla T)+\vec{v} \cdot \nabla T+A\left(S r(T)-C_{0}\left(T-T_{a}\right)\right), \\
& \frac{d S}{d t}=-C_{S} S r(T),
\end{aligned}
$$

where the reaction rate is a modified Arrhenius rate,

$$
r(T)=e^{-B /\left(T-T_{a}\right)} .
$$




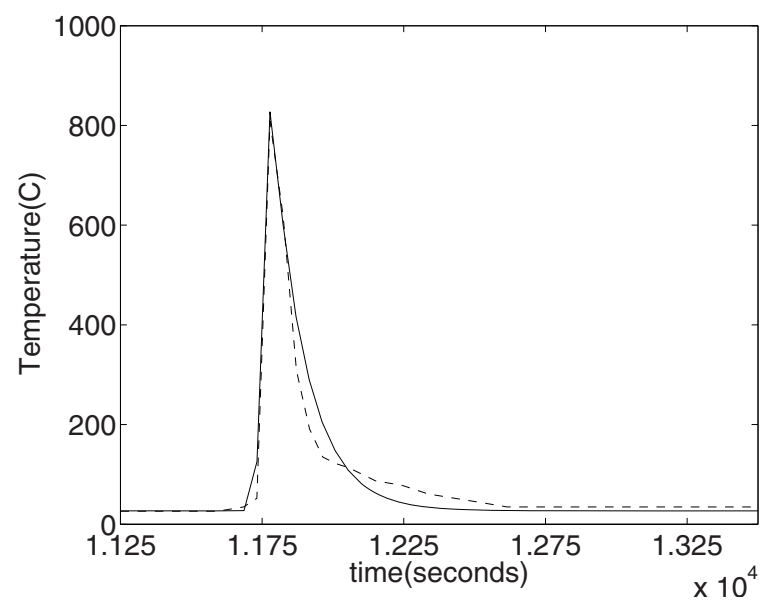

Fig. 1. Measured time-temperature profile (dotted line) in a wildland fire at a fixed sensor location, digitized from [1, and computed profile (solid line) 2]. Coefficients of the model were identified by optimization to match to measured profile.

Eq. (1) represents a 2D balance of energy in a fire layer of some unspecified finite vertical thickness, (2) represents the balance of fuel, $T$ is the temperature of the fire layer, $r(T) \in[0,1]$ is the reaction rate, assumed to depend only on the temperature, $S \in[0,1]$ is the fuel supply mass fraction (the relative amount of fuel remaining), $k$ is the diffusion coefficient, $A$ is the temperature rise per second at the maximum burning rate with full initial fuel load and no cooling present, $B$ is the proportionality coefficient in the modified Arrhenius law, $C_{0}$ is the scaled coefficient of the heat transfer to the environment, $C_{S}$ is the fuel relative disappearance rate, $T_{a}$ is the ambient temperature, and $\vec{v}$ is the given wind speed from the atmosphere.

Physics-based models of the form similar to (11]3) are known [345]. The system (1, 2) with the reaction rate (3) is formally the same as combustion equations of premixed fuel, with cooling added. Our interpretation is that the equations are a rough approximation of the aggregated behavior of small-scale random combustion. We are going to add variables and equations only as needed to match the observed fire behavior. Possible future extension include pyrolysis, multiple fuels, crown fire, and moisture (treated as a fuel with negative heat content).

The reaction rate (3) has been modified to be exactly zero at ambient temperature (according to chemical kinetics, the reaction rate should be zero only at absolute zero temperature); consequently equations (1.3) admit traveling wave solutions. The temperature in the traveling wave has a sharp leading edge, followed by an exponentially decaying cool-down trailing edge (Fig. 1). The wave speed can be found numerically [5], but no mathematical proof of the existence of traveling waves and their speed for system (113) seems to be known.

Since weather is a very significant influence on fire and fire in turn has influence on weather, coupling of the fire model with the Weather Research Forecast Model 
(WRF) 677] is in progress [8]. It is now well established that fire dynamics can be understood only by modeling the atmosphere and the fire together, e.g., [9].

\section{Coefficient Identification}

The reason for writing the coefficients of model (1] 3) in that particular form is that it is basically hopeless to use physical coefficients and expect a reasonable solution. The fuel is heterogeneous, the fire blends into the atmosphere, and it is unclear what exactly are, e.g., the diffusion and the heat loss coefficients. Instead, we consider information that can be reasonably obtained. For example, consider the heat balance term at full fuel load $(S=1)$,

$$
f(T)=r(T)-C_{0}\left(T-T_{a}\right) .
$$

Generally, there are three temperatures which cause $f(T)$ to be zero (where the heat created by the reaction is balanced by the cooling): $T_{a}$, arising from our modification of the Arrhenius rate (otherwise there is one zero just above $T_{a}$ because some reaction is always present); the autoignition temperature; and finally the "high temperature regime" [10, which is the hypothetical steady burning temperature assuming fuel is constantly replenished. Substituting reasonable values for the latter two temperatures allows us to determine reasonable approximate values of the coefficients $B$ and $C_{0}$. Assuming zero wind, we can then determine the remaining coefficients in 1D dynamically from a measured temperature profile in an actual wildland fire [1]. Reasonable values of the remaining coefficients are obtained by (i) passing to a nondimensional form and matching nondimensional characteristics of the temperature profile, such as the ratio of the widths of the leading and the trailing edge and (ii) using the traveling wave speed and intensity to match the scale. Once reasonable starting values are known, the coefficients can be further refined by optimization to match the measured temperature profile (Fig. 1). See 2] for details. Identification of coefficients in the presence of wind in the model is in progress [11.

\section{Numerical Solution}

The coefficients identified from a 1D temperature profile were used in a 2D model, discretized by standard central finite differences with upwinding of the convection term. Implicit time stepping by the trapezoidal method (Crank-Nicholson) was used for stability in the reaction term and to avoid excessively small time steps. The large sparse system of linear equations in every time step was solved by Newton's method with GMRES as the linear solver, preconditioned by elimination of the fuel variables (which are decoupled in space) and FFT. One advantage of this approach is that even after more fuels are added, the fuel variables can still be eliminated at every node independently, and the resulting system has the same sparsity structure as the heat equation alone. Typically, mesh steps of $1 \mathrm{~m}$ $-5 m$ and time steps of $1 s-5 s$ are required to resolve the moving fire front and to get a reasonable simulation [11]. 


\section{Data Assimilation}

Data assimilation is how the data is actually injected into a running model. We have chosen a probabilistic approach to data assimilation, which is common in geosciences [12. The ensemble filter method used here is one possible approach. This method estimates the system state from all data available by approximating the probability distribution of the state by a sample, called an ensemble. The ensemble members are advanced in time until a given analysis time. At the analysis time, the probability density, now called the prior or the forecast, is modified by accounting for the data, which are considered to be observed at that time. This is done by multiplying the prior density by data likelihood and normalizing (the Bayesian update). The new probability density is called the posterior or the analysis density. The data likelihood is a function of state. It is equal to the density of the probability that the data could have been obtained for the given state. It can be found from the probability density of data error, which is assumed to be known (every measurement must be accompanied by an error estimate or it is meaningless), and an observation function (sometimes called the forward operator in an inverse problem context). The argument of the observation function is a model state and its value is what the correct value of the data should be for that state.

The Ensemble Kalman Filter (EnKF) [13]14 works by forming the analysis ensemble as linear combinations of the forecast ensemble, and the Bayesian update is implemented by linear algebra operating on the matrix created from ensemble members as columns.

In a wildland fire application, EnKF produces nonphysical states that result in instability and model breakdown [2[15. The linear combination of several states with a fire in slightly different locations cannot match data with a fire in another location. In a futile attempt to match the data, EnKF makes the analysis ensemble out of crazy linear combinations of the forecast ensemble. These can be fatal immediately. Even if they are not, the analysis ensemble tends to bear little resemblance to the data. This can be ameliorated to some degree by penalization of nonphysical states 2[15 but not well enough to produce a reliable method: the filter is capable of only a small adjustment of the fire location, and its parameters have to be finely tuned for acceptable results, especially the data variance is required to be artificially large.

For this reason, we have developed a new method that updates the location of firelines directly, called the morphing ensemble filter [16]17, which is based on techniques borrowed from registration in image processing. Given two images as pixel values on the same domain, the registration problem is to find a mapping of the domain that turns one of the given images into the other with a residual (the remaining difference) that is as small as possible. We write this mapping as the identity plus a registration mapping. The registration mapping should be as small and as smooth as possible. Hence, one natural way to solve the automatic registration problem is by optimization, and we use a method based on [18. Once the registration mapping and the residual are found, we can construct intermediate images between the two images by applying the registration mapping 


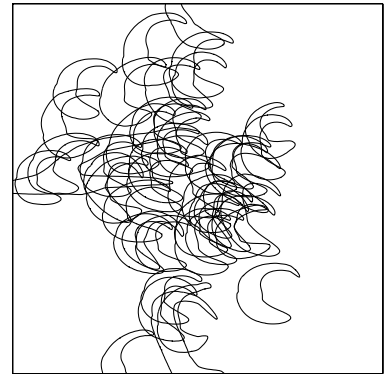

(a)

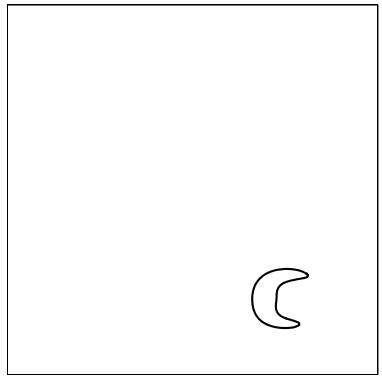

(b)

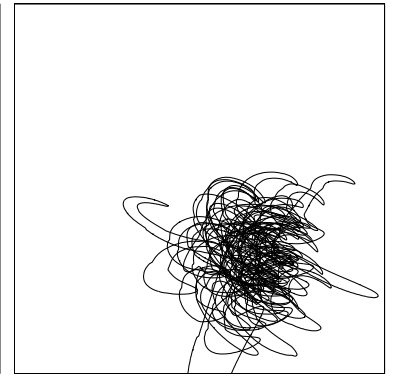

(c)

Fig. 2. Data assimilation by the morphing ensemble filter [16]. Contours are at $800 \mathrm{~K}$, indicating the location of the fireline. The reaction zone is approximately inside the moonshaped curve. This fireline shape is due to the wind. The forecast ensemble (a) was obtained by a perturbation of an initial solution. The fire in the simulated data (b) is intentionally far away from the fire in the initial solution. The data for the EnKF engine itself consisted of the transformed state obtained by registration of the image. The analysis (c) shows the adjusted location of the fire in the analysis ensemble members.

and adding the residual, both multiplied by some number between zero and one. Generalizing this observation, we can choose one fixed state with a fire in some location, and register all ensemble members. The registration mappings and the residuals then constitute a transformed ensemble. The morphing ensemble filter consists of applying the EnKF to the transformed ensemble and thus using intermediate states rather than linear combinations. This results in an adjustment of both the intensity and the position of the fire. Now the fireline in the data can be anywhere in the domain, and the data variance can be small without causing filter divergence.

Fire data in the form of an image can be assimilated by registering the image and then using the transformed image as the observation. The observation function is then only a pointwise transformation of the model state to gridded values and interpolation. In the general case, the transformed observation function is defined by composition of functions, and so it is a highly nonlinear function of the registration mapping. So, assimilation of weather station data and sensor data will be more complicated and can result in strongly non-Gaussian multimodal probability distributions. For example, a sensor can read low temperature either because the fire did not get there yet or because it has already passed, so the analysis density after assimilating one sensor reading would be concentrated around these two possibilities.

Another view is that the morphing EnKF works by transforming the state so that after the transformation, the probability distribution is closer to Gaussian. We have also developed another new technique, called predictor-corrector filters [17, to deal with the remaining non-Gaussianity. 


\section{Data Acquisition}

Currently, we are using simple simulated data, as described in the previous section. In the future, data will come from fixed sensors that measure temperature, radiation, and local weather conditions. The fixed Autonomous Environmental Sensors (AESs), positioned so as to provide weather conditions near a fire, are mounted at various heights above the ground on a pole with a ground spike (Fig. [3). This type of system will survive burnovers by low intensity fires. The temperature and radiation measurements provide a direct indication of the fire front passage and the radiation measurement can also be used to determine the intensity of the fire [20]. The sensors transmit data and can be reprogrammed by radio.

Data will come also from images taken by sensors on either satellites or airplanes. Three wavelength infrared images can then be processed using a variety of algorithms to extract which pixels contain a signal from fire and to determine the energy radiated by the fire and even the normal velocity of the fireline (Fig. 3b).

The data is related to the model by an observation function. Currently, we are using simple simulated observation functions, as described in the previous section. Our software framework supports using multiple types of observation functions (image, weather station, etc.). Each data item must carry information about which observation function is to be used and any requisite parameters (coordinates, scaling factors, etc.) in metadata.

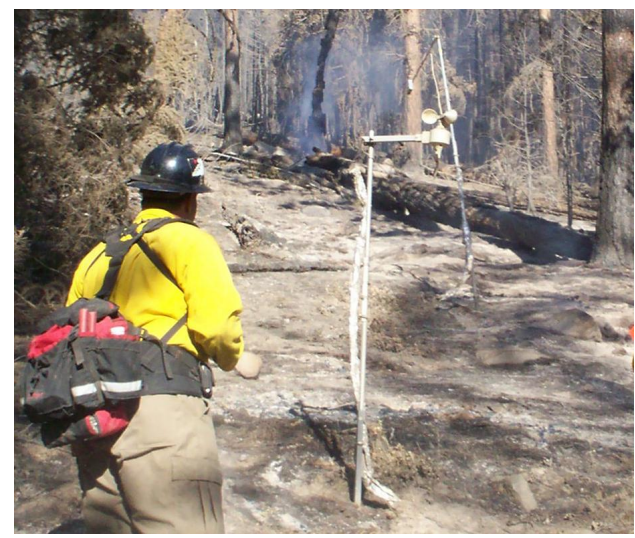

(a)

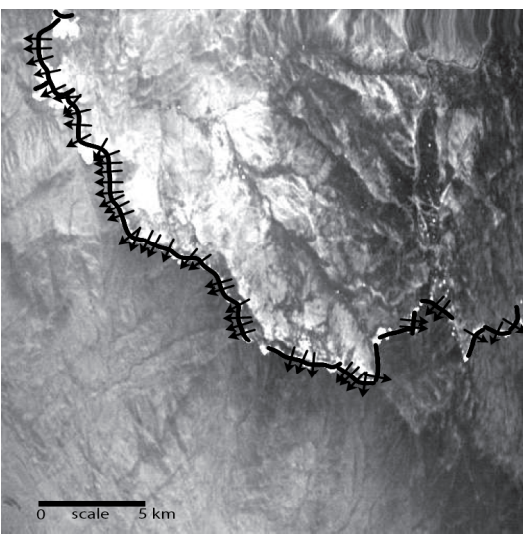

(b)

Fig. 3. (a) AES package deployed on the Tripod Fire in Washington (2006). The unit shown has been burned over by the fire but is still functional. The datalogger package is buried a small distance beneath the surface to shield it from the effects of the fire. (b) Airborne image processed to extract the location and propagation vector of the fireline (reproduced from 19 by permission). 


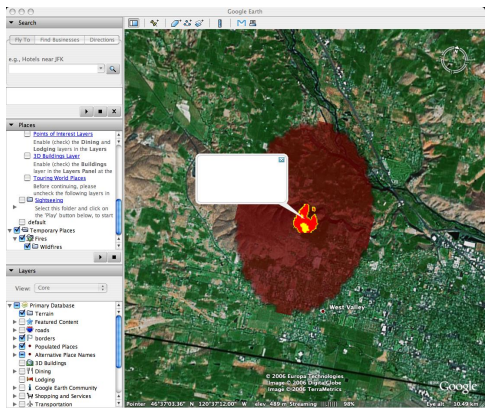

(a)

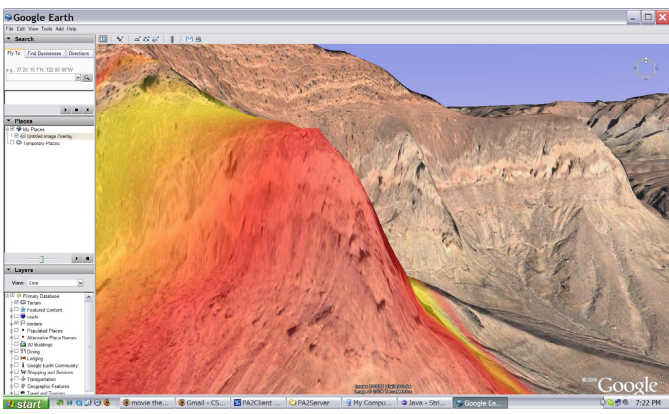

(b)

Fig. 4. The Google Earth Fire Layering software tool. (a) Closeup on a single fire (b) $3 \mathrm{D}$ view.

\section{Visualization}

Our primary tool for visualization is storing gridded data from the model arrays into files for visualization in Matlab. Our Google Earth Fire visualization system (Fig. 4) greatly simplifies map and image visualization and will be used for model output in the future. The user can control the viewing perspective, zooming into specific sites, and selecting the time frame of the visualization within the parameters of the current available simulation. Google Earth is quickly becoming a de-facto standard and wildland fire visualizations in Google Earth are now available from several commercial and government sources, e.g., 2122.

\section{Acknowledgment}

This research has been supported by the National Science Foundation under grants CNS 0325314, 0324989, 0324988, 0324876, and 0324910.

\section{References}

1. Kremens, R., Faulring, J., Hardy, C.C.: Measurement of the time-temperature and emissivity history of the burn scar for remote sensing applications. Paper J1G.5, Proceedings of the 2nd Fire Ecology Congress, Orlando FL, American Meteorological Society (2003)

2. Mandel, J., Bennethum, L.S., Beezley, J.D., Coen, J.L., Douglas, C.C., Franca, L.P., Kim, M., Vodacek, A.: A wildfire model with data assimilation. CCM Report 233, http://www.math.cudenver.edu/ccm/reports/rep233.pdf (2006)

3. Asensio, M.I., Ferragut, L.: On a wildland fire model with radiation. International Journal for Numerical Methods Engineering 54 (2002) 137-157

4. Grishin, A.M.: General mathematical model for forest fires and its applications. Combustion Explosion and Shock Waves 32 (1996) 503-519 
5. Weber, R.O., Mercer, G.N., Sidhu, H.S., Gray, B.F.: Combustion waves for gases $(L e=1)$ and solids $(L e \rightarrow \infty)$. Proceedings of the Royal Society of London Series A 453 (1997) 1105-1118

6. Michalakes, J., Dudhia, J., Gill, D., Klemp, J., Skamarock, W.: Design of a nextgeneration weather research and forecast model. Towards Teracomputing: proceedings of the Eighth Workshop on the Use of Parallel Processors in Meteorology, European Center for Medium Range Weather Forecasting, Reading, U.K., November 16-20, 1998. ANL/MCS preprint number ANL/MCS-P735-1198 (1998)

7. WRF Working Group: Weather Research Forecasting (WRF) Model. http://www.wrf-model.org (2005)

8. Beezley, J.D.: Data assimilation in coupled weather-fire models. Ph.D. Thesis, in preparation (2008)

9. Coen, J.L.: Simulation of the Big Elk Fire using using coupled atmosphere-fire modeling. International Journal of Wildland Fire 14 (2005) 49-59

10. Frank-Kamenetskii, D.A.: Diffusion and heat exchange in chemical kinetics. Princeton University Press (1955)

11. Kim, M.: Numerical modeling of wildland fires. Ph.D. Thesis, in preparation (2007)

12. Kalnay, E.: Atmospheric Modeling, Data Assimilation and Predictability. Cambridge University Press (2003)

13. Evensen, G.: Sequential data assimilation with nonlinear quasi-geostrophic model using Monte Carlo methods to forecast error statistics. Journal of Geophysical Research 99 (C5) (1994) 143-162

14. Houtekamer, P., Mitchell, H.L.: Data assimilation using an ensemble Kalman filter technique. Monthly Weather Review 126 (1998) 796-811

15. Johns, C.J., Mandel, J.: A two-stage ensemble Kalman filter for smooth data assimilation. Environmental and Ecological Statistics, in print. CCM Report 221, http://www.math.cudenver.edu/ccm/reports/rep221.pdf (2005)

16. Beezley, J.D., Mandel, J.: Morphing ensemble Kalman filters. CCM Report 240, http://www.math.cudenver.edu/ccm/reports/rep240.pdf (2007)

17. Mandel, J., Beezley, J.D.: Predictor-corrector and morphing ensemble filters for the assimilation of sparse data into high dimensional nonlinear systems. 11th Symposium on Integrated Observing and Assimilation Systems for the Atmosphere, Oceans, and Land Surface (IOAS-AOLS), CD-ROM, Paper 4.12, 87th American Meterological Society Annual Meeting, San Antonio, TX, January 2007.

18. Gao, P., Sederberg, T.W.: A work minimization approach to image morphing. The Visual Computer 14 (1998) 390-400

19. Ononye, A.E., Vodacek, A., Saber, E.: Automated extraction of fire line parameters from multispectral infrared images. Remote Sensing of Environment (to appear)

20. Wooster, M.J., Zhukov, B., Oertel, D.: Fire radiative energy for quantitative study of biomass burning: derivation from the BIRD experimental satellite and comparison to MODIS fire products. Remote Sensing of Environment 86 (2003) 83-107

21. NorthTree Fire International: Mobile Mapping/Rapid Assessment Services. http://www.northtreefire.com/gis/ (2007)

22. USGS: RMGSC - Web Mapping Applications for the Natural Sciences. http://rockyitr.cr.usgs.gov/rmgsc/apps/Main/geiDownloads.html (2007) 